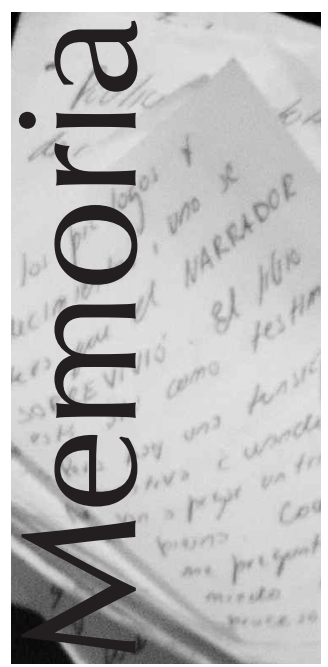

\title{
Balance epidemiológico argentino
}

\author{
An epidemiological assessment \\ of Argentina
}

Carrillo, Ramón ${ }^{1}$

RESUMEN Este texto de Ramón Carrillo (1906-1956) corresponde al discurso de apertura de la $2^{\text {a }}$ Conferencia de Epidemiología y Endemias, del 6 de octubre de 1947. Fue publicado originalmente en 1949 en el libro Política sanitaria argentina, que reúne artículos, conferencias y discursos oficiales de quien, en 1946, decidió asumir el cargo de Secretario de Salud Pública y, posteriormente, fue el primer Ministro de Salud Pública que tuvo la Argentina. En palabras del propio autor: "uno de los triunfos de la medicina contemporánea reside, precisamente, en haber resuelto -o casi resuelto- los graves problemas de las enfermedades infecciosas y que si aún siguen imperando en algunos aspectos se debe pura y exclusivamente a la despreocupación de los gobiernos por las cosas que atañen directamente a la vida y a la salud del pueblo"; lo que no hace más que recordarnos el carácter social de los problemas de salud, enfermedad y atención de los conjuntos sociales. La transcripción de este discurso en la sección "Memoria e historia" tiene el propósito de destacar la vigencia de un pensamiento sanitario formulado hace 65 años, que interpela la realidad sanitaria argentina actual.

PALABRAS CLAVE Historia del Siglo XX; Políticas Públicas de Salud; Epidemiología; Enfermedades Trasmisibles; Desigualdad en Salud; Medicina Social; Argentina.

ABSTRACT This text is the transcription of Ramon Carrillo's opening speech at the 2nd Conference of Epidemiology and Endemic Diseases on October 6, 1947. It was originally published in 1949 in the book Política sanitaria argentina (Argentine public health policy), which includes articles, conferences and official speeches by this man who, in 1946, took office as Public Health Secretary and later became the first Public Health Minister of Argentina. In the words of the author himself: "one of the triumphs of contemporary medicine resides precisely in that it has solved or almost solved - the serious problems of infectious diseases; if these diseases continue to persist in certain aspects it is due exclusively to government negligence regarding the things that directly affect the life and health of the people." Such words serve to remind us of the social character of the health-disease-care problems in social groups. The reprinting of this speech in the section "Memory and History" seeks to highlight the relevance of public health thought formulated 65 years ago that continues to speak to the current situation of public health in Argentina.

KEY WORDS History, 20th Century; Health Public Policy; Epidemiology; Communicable Diseases; Health Inequality; Social Medicine; Argentina.

\footnotetext{
${ }^{1}$ Médico neurocirujano. Primer Ministro de Salud Pública de la República Argentina. (1906-1956).
} 
En las encrucijadas se plantea el problema de cuál es el rumbo y cuál es el camino que conduce más directamente a destino; en el instante de la opción es fundamental no ilusionarse por el horizonte luminoso y fácil; es preferible tomar por la ruta más escarpada si ello fuera necesario para evitar la contingencia del error.

En los grandes asuntos de la epidemiología estamos en un momento geométrico de encrucijadas. No ilusionarnos y no equivocar el camino.

Viven aún médicos e higienistas que asistieron a las revolucionarias transformaciones de la bacteriología y de la epidemiología de fin del siglo pasado. Ayer no más la doctrina de los miasmas servía para explicar la difusión de las enfermedades infecciosas; muchos capítulos de epidemiología, a principios de este siglo, estaban todavía impregnados de las afirmaciones hipocráticas que durante 24 siglos fueron palabra sagrada en la medicina.

\section{LA IDENTIFICACIÓN DE LOS AGENTES DE LAS ENFERMEDADES}

Pero solo en 50 años la epidemiología superó el progreso de 2.400 años, al identificar uno por uno los agentes productores de cada enfermedad y descubrir el suero o la vacuna que la cura o la previene, desapareciendo prácticamente las grandes plagas pestilenciales que diezmaron a la humanidad desde los tiempos bíblicos, el cólera, la peste, la fiebre amarilla y tantas otras.

La medicina había encontrado un camino fecundo en la inmunología y cuando parecía llegarse al fin del mismo surgen, como detrás de una colina imprevista en el paisaje, los antibióticos, tales como la penicilina y la estreptomicina, ofreciendo posibilidades sin límite y un horizonte infinito. No es aventurado afirmar que se abre una nueva era y que conviene efectuar un rápido reajuste técnico de nuestras organizaciones sanitarias para obtener el máximo rendimiento de estas flamantes adquisiciones de la ciencia médica y de los planteos más recientes de la biología.

\section{LAS EPIDEMIAS Y ENDEMIAS}

La lucha contra las enfermedades infecciosas tiene algo de arte militar. El invento de un arma nueva impone tácticas ofensivas y defensivas también nuevas, y de una experiencia bélica a otra los instrumentos de destrucción parecen pueriles comparados con los anteriores. Así ocurre también en la guerra contra las epidemias y endemias.

Es tan rápido el progreso que las ideas envejecen casi en el mismo momento en que son concebidas. Behring, el creador del suero antidiftérico, brinda a la humanidad su remedio que permite salvar a miles y miles de niños, pero al mismo tiempo anuncia melancólicamente, casi como una profecía, que ese suero descubierto por él no resuelve el problema de la difteria y que alguien superaría el procedimiento aplicando la vacunación activa y él mismo, en 1911, formula un sistema de vacunación que se emplea hasta el descubrimiento de las anatoxinas -es decir-aquello que Behring profetizó con 25 años de antelación.

La patología de las enfermedades infecciosas ofrece dos aspectos: uno el individual y otro el colectivo. El problema del caso individual es el punto de vista estrictamente científico y de la investigación clínica o terapéutica; es allí, el escarbar diario de los hechos de observación, de donde extraemos los materiales para el progreso de la clínica de las enfermedades infecciosas. El problema colectivo, es decir, la consideración de las enfermedades epidémicas y endémicas desde el punto de vista social, encuadradas como materia del Estado es el que me interesa promover ante ustedes como Secretario de Salud Pública de la Nación para pedirles su inestimable consejo y concretar las medidas adecuadas para proteger a nuestra población de ciertos males que se adentran y nos quitan vidas de trabajadores argentinos todos los días, ensañándose contra nuestras clases rurales no pudientes, mal defendidas y peor educadas sanitariamente.

Para esbozar ante ustedes nuestras reflexiones y preocupaciones cotidianas sobre estos problemas, voy a intentar un balance sanitario, sobre el estado de nuestro país en el terreno de la patología infecciosa y al entrar en él, lo hago con el exclusivo propósito de escuchar más tarde vuestra autorizada palabra. 


\section{BALANCE SANITARIO ARGENTINO}

Puede fijarse el año 1910 como el punto culminante en el progreso de la ciencia inmunológica, luego sigue un período estacionario y finalmente en 1935 se inicia el período de los antibióticos.

El año 1910 es para la Argentina un punto de reparo muy importante, porque desde ese año se comienza a liquidar con el pasado en materia de enfermedades infecciosas y por eso lo tomaremos como base para nuestras referencias estadísticas y demográficas.

En ese año todavía la viruela y la fiebre tifoidea, por ejemplo, hacían estragos no solo en el interior del país, sino también en importantes barrios de la Capital. Poco a poco se ha tomado el control de esas y otras enfermedades infecciosas a punto tal de hacerlas desaparecer del mapa epidemiológico; pero los resultados no son todavía satisfactorios, pues algunas enfermedades que deberían no existir más, aún siguen haciendo estragos, debido un tanto a la falta de organización y de poderío de la sanidad argentina y también por la ausencia de cultura y educación sanitaria, ya que la ignorancia, la suciedad, la subalimentación y la vivienda antihigiénica son los auténticos caldos de cultivo de los gérmenes microbianos; bajo ese punto de vista, la salud pública es un problema de orden cultural y educacional.

Las enfermedades infecciosas de la infancia en el año 1910 representaban el 25\% del total de las causas de muerte; se reducen a menos del $10 \%$ en la iniciación de la era de los antibióticos, es decir en 1935. La difteria que producía una mortalidad de 72 por cada 100 mil habitantes en edades comprendidas entre 1 y 14 años, baja su tasa a 9, lo cual significa un descenso de un $88 \%$. La mortalidad por sarampión, en esas mismas edades, que era de 27 , baja a 6 , es decir señala una caída de $77 \%$. La escarlatina en esas mismas edades y fechas, baja de 27 a 7 , comprobándose un descenso de $73 \%$. La coqueluche, baja de 20 a 5, lo cual significa un descenso de un $73 \%$. La mortalidad por tuberculosis en todas las edades que nos daba un índice calculado de 224 por 100 mil de población, ya ha descendido a menos de 100 para todo el país, y se aproxima en la Capital Federal al índice basal de
70, con lo cual nos plantea el problema de que esa dolencia dejará de ser, si se toman las medidas adecuadas, una enfermedad social para transformarse en una endemia.

La neumonía que pareció ser refractaria a la acción inmunológica por la gran variedad de antígenos concurrentes, desciende su índice de mortalidad en un $30 \%$ en los 15 años que van de 1920 a 1935 . La bronconeumonía tiene un descenso de un $25 \%$ en ese mismo lapso.

Las enfermedades puerperales, que ocasionaban una mortalidad de 34 mujeres por cada 100 mil habitantes en 1910, baja a 17 en 1935, lo que equivale decir que se le quitó a la muerte un $50 \%$ del numeroso grupo que ya había tomado entre sus manos. Las enteritis graves en todas las edades baja su mortalidad en un $85 \%$; y la fiebre tifoidea desciende el $90 \%$ en el lapso que estamos considerando.

En síntesis, puede mostrarse con satisfacción el balance sanitario arriba transcripto, y es de presumir que al extenderse la era de los antibióticos y de la profilaxis bien organizada por el Estado, prácticamente desaparecerán de nuestro país una enorme cantidad de factores de muerte, como ha ocurrido en otras partes del mundo, y ello será un motivo de orgullo para nuestra civilización y cultura nacional.

La poliomielitis, la brucelosis y otras enfermedades endemoepidémicas que aún preocupan a las autoridades por no tener un camino trazado ni una solución definitiva, no creo que se mantengan por mucho tiempo como problemas, pues los ingentes esfuerzos técnicos y científicos, tanto en nuestro país como en el extranjero, Ilevarán fatalmente a un desenlace favorable.

Uno de los triunfos de la medicina contemporánea reside, precisamente, en haber resuelto -o casi resuelto- los graves problemas de las enfermedades infecciosas y que si aún siguen imperando en algunos aspectos se debe pura y exclusivamente a la despreocupación de los gobiernos por las cosas que atañen directamente a la vida y a la salud del pueblo. Un signo halagador de este triunfo de la medicina se advierte al estudiar el promedio de vida del hombre actual. En nuestro país el hombre vivía, como término medio en el año 1900, 42 años. Hoy, al salvarse millares de vidas que antes se perdían irremediablemente, estamos Ilegando a un promedio de vida de 60 
años, cifra por supuesto inferior a los 67 alcanzados en otros países. Hemos prolongado la vida del hombre al reducir en forma impresionante la mortalidad por enfermedades infectocontagiosas.

La diferencia de 20 años que señalamos actualmente entre los 60 años promedio de vida en 1947, y los 40 años de promedio en 1900, se origina principalmente en la reducción de la mortalidad infantil.

A principios de este siglo el número de niños muertos entre los 0 y 5 años era tan grande, que solo en esa época de la vida el país perdía tanto caudal humano como entre los 5 y los 100 años de edad. Hoy el parámetro de máxima destrucción de vidas humanas se ha desplazado en nuestro país en un $50 \%$ a la edad de 60 años. En otros términos podríamos decir, esquematizando, que la mitad de los fallecimientos se producen entre los 0 y 5 años y la otra mitad entre los 50 y 60 años; quedaría un porcentaje mínimo entre ambos extremos de la vida.

Las estadísticas nos señalan por otra parte que del total de fallecimientos por enfermedades infecciosas, la mayoría se producen en los medios rurales, donde la profilaxis y el tratamiento de las mismas no están organizados como en nuestros medios urbanos.

\section{ESTADO SANITARIO DE LA CAMPAÑA ARGENTINA}

La lucha sanitaria debe dirigirse intensamente a extinguir los focos endémicos argentinos en el interior del país, es decir, terminar de alguna manera con el paludismo, con la anquilostomiasis, con las infecciones puerperales, con las enteritis endémicas y epidémicas y con las afecciones infectocontagiosas. Los índices nos señalan que la mortalidad infantil en todas las edades es enormemente superior en la campaña si se la compara con la de las ciudades argentinas. La cifra de niños y adolescentes muertos en nuestro país crece en progresión casi vertical en cuanto salimos de las grandes urbes y nos dirigimos hacia el norte o hacia el sud, recorriendo los fértiles campos de la patria; lo mismo aumenta el número de madres fallecidas en o después del parto, de modo tal que la mortalidad maternal rural es un tercio más alta que en las grandes ciudades.

He ahí planteado nuestro gran problema sanitario nacional -y que deberá preocuparnos hondamente- puesto que las reservas biológicas de la Nación están justamente en los medios rurales, a los que a veces les negamos tantas cosas. La llamada provincia "pobre" de Santiago del Estero, que más que pobre es "olvidada"; con un quinto de la población de la Capital Federal, entrega a la patria tantos nacimientos -es decir, otros tantos hijos- como todos los que produce la populosa y rica ciudad de Buenos Aires. Más de la mitad de los niños argentinos nacen en comunidades rurales y son ellas las que alimentan y enriquecen -por la arrolladora migración interna- las zonas urbanas y la mano de obra de la industria nacional. La ciudad de Buenos Aires y Rosario ya se habrían despoblado si no se produjera el fenómeno casi inevitable del éxodo de la campaña a la ciudad, puesto que el número de nacimientos en estas ciudades no alcanzan a cubrir los claros que se producen por defunciones en los centros urbanos.

Si conseguimos elevar el standard sanitario de los niños de nuestra campaña, aseguraremos al mismo tiempo el mínimo indispensable de obreros para la industrialización del país, proceso que se encuentra por ahora en las grandes ciudades y compensaríamos quizás -en parte- el fenómeno tan grave de la despoblación de la campaña.

La neumonía, la gripe, las enteritis graves, la tifoidea y salmonelosis, la difteria y el sarampión, escarlatina y coqueluche, perseguidas implacablemente por los médicos y las medidas preventivas en las ciudades, ha ido a enseñorearse en nuestros niños del interior; allí debemos dirigir los esfuerzos para nivelar, siquiera en parte, el desequilibrio sanitario y demográfico de la República.

La tuberculosis y la lúes desempeñan con respecto a los adultos, en los medios rurales, el mismo papel destructivo que las enfermedades que señalamos como típicas en la infancia, y habría más de un sorprendido si diéramos a conocer los detalles observados en esa materia por nuestros delegados sanitarios en el Chaco, Formosa, Misiones, o Corrientes. Los índices de morbilidad y mortalidad rural en el momento 
actual en nuestro país, demuestran que tenemos una pérdida de vidas humanas en la campaña tan caudalosa como la que sufría la Nación entera en el año 1900, cuando el saneamiento rural, los sueros y vacunas y la penicilina eran desconocidos.

\section{LAS DELEGACIONES SANITARIAS}

Más de tres millones de argentinos viven en comunidades rurales carentes de toda organización sanitaria y asistencial, lo que supone que una quinta parte de la población de nuestra patria se encuentra en la prehistoria de la higiene y de la medicina social. Hemos constituido con ingentes sacrificios nuestras delegaciones sanitarias en el interior del país, siempre luchando con dificultades materiales y de toda índole, pero estamos en camino de resolver esta situación y la resolveremos si Dios y los tiempos nos ayudan.

No es menester forzar los cálculos para tener una idea aproximada de cuánto costaría un plan sanitario que termine con el estado de cosas que he diseñado sobriamente y sin emplear la pintura intensa a que se presta el asunto de por sí, profundamente dramático y angustioso. La Municipalidad de la Capital dentro de las limitaciones que le impone su presupuesto invierte $\$ 20$ por año y por habitante para ofrecer los servicios de profilaxis y de asistencia médico-social. Estados Unidos tiene el propósito de invertir solamente en la lucha contra las enfermedades venéreas 4 dólares por habitante. Las mutualidades mejor organizadas del país invierten $\$ 40$ por socio y por año al solo fin del tratamiento médi$\mathrm{Co}$, sin subsidio de enfermedad ni ningún otro servicio médico-social.

Si invirtiéramos en todo el país la misma cantidad de dinero por habitante que invierte actualmente la Municipalidad de Buenos Aires, es decir, \$20 por habitante y por año, llegaríamos a la conclusión de que el presupuesto de Salud Pública debería ser de 320 millones, es decir, el doble del actual. Naturalmente que todos saben que $\$ 20$ por año y por habitante en la Capital apenas alcanza para mantener -y mal mantenidos- los servicios médicos de la ciudad, pero la cifra que señalamos es lo que costaría nada más que para colocar el estado sanitario rural al mismo nivel que el de la Capital. Sin embargo, siempre sería económico, puesto que en Estados Unidos se calcula que las pérdidas por enfermedad ascienden a 40 mil millones de pesos por año; teniendo nosotros la décima parte de la población de Estados Unidos -y suponiendo que nuestra organización sanitaria fuera igual en eficacia a la de ese país- podríamos calcular nuestras pérdidas anuales por enfermedad en 4 mil millones de pesos, dato que coincide con estudios efectuados por médicos argentinos que aprecian nuestro déficit, solamente por tuberculosis, en 300 millones de pesos por año.

Estas cifras ofrecen idea aproximada pero gráfica de cómo incide sobre la economía de un país su estado sanitario apreciado en pesos moneda nacional, al gravitar directamente sobre lo que en la economía de un país se Ilama la productividad del trabajo.

Para tranquilidad de nuestra conciencia debemos manifestar que esta situación no es específicamente argentina, sino de casi todos los países americanos que, en general, cuentan con una sanidad mejor organizada que la nuestra y si no hacen más es solo falta de recursos. Nosotros podríamos disponer de los recursos, pero en cambio carecemos de una buena organización que recién ahora se está concretando en un lento y penoso esfuerzo destinado a centralizar paulatinamente los servicios médico-sanitarios y médico-sociales.

Mientras el sistema de previsión social organiza la protección de la salud de las clases no pudientes y las organiza sobre la contribución de los beneficiarios, comenzando de abajo para arriba, el Estado debe seguir actuando de arriba para abajo por medio de los organismos técnicos de que disponemos, vigorizándolos y extendiendo su acción como corresponde. Solo por un movimiento convergente desde el individuo al Estado y desde el Estado al individuo se podrían resolver y financiar racionalmente las cuestiones atinentes con la salud pública, y no es difícil que en diez años más estos planos se superpongan en un solo sistema de asistencia somática, mental y social, preventiva y curativa, profiláctica y terapéutica. 


\section{BASES DE UN PLAN SANITARIO INMEDIATO}

No es necesario inventar nada nuevo para conseguir un descenso de los índices de morbimortalidad por enfermedades infecciosas. Los mismos principios permitieron reducir a cero las enfermedades pestilenciales y a un $10 \%$ las endemias comunes, pueden ser perfectamente adecuados a un programa simple y práctico de eficacia inmediata. Ya lo hemos presentado a ese plan con mayores detalles en nuestro plan quinquenal de salud pública, pero quisiera destacar ante ustedes los elementos básicos de ese plan en lo que se vincula con las enfermedades infectocontagiosas.

a) La organización previa de la estadística sanitaria y una exacta discriminación de las causas de muerte. En este terreno -como en casi todos- nuestro país carecía de estadísticas precisas y sin estadísticas precisas no se puede planificar ninguna acción eficaz. El certificado confidencial de la causa de muerte permitirá "sincerar" las tablas de mortalidad y morbilidad en la Argentina. Recientemente realizamos experimentalmente un censo sanitario familiar tomando sesenta manzanas de la Capital Federal; sus resultados no son para ser publicados, pues descubrirían una vergüenza nacional, difícil de atenuar, por más que se adujeran características de miseria, ignorancia y abandono del barrio elegido para la encuesta. Ese barrio de la Capital Federal está en iguales o peores condiciones médico-sociales que las tolderías de indios en Formosa.

b) Creación de una red de laboratorios destinados al diagnóstico precoz de todas las enfermedades infectocontagiosas, empezando por los test serológicos comunes, y los procedimientos corrientes de identificación microbiana, de los cuales carece el interior del país e incluso carece hasta de técnicos preparados para estas tareas. A fin de año habilitaremos ya los Laboratorios Central de Catamarca y San Luis.

c) Educación sanitaria de la población. Si las campañas de difusión de los conocimientos sanitarios se abordan mal, resulta algo muy costoso. La propaganda sanitaria debe ser barata, directa y eficaz. Para ellos hemos creado el Instituto de Educación Sanitaria que dentro de pocos días entrará en acción. El mejor procedimiento sería utilizar la vía natural de las maestras y maestros para hacer llegar hasta los hogares las normas de higiene familiar, a través del niño que concurre a la escuela. La sanidad podría tener 40 mil agentes entre los maestros que bien instruidos completarían la tarea civilizadora aportando un nuevo elemento de cultura como es la de poner en manos de las masas una serie de principios e ideas que las ayuden a vivir en salud.

d) La instalación de unidades sanitarias rurales será también pronto una realidad. Estará integrada por un médico, un dentista, una partera y enfermeras de salud pública o visitadoras sanitarias, que serán ni más ni menos, que agentes del poder público extendido cordialmente para proteger a la familia rural.

e) La extensión y ampliación de las obras sanitarias, las obras de saneamiento e ingeniería sanitaria para las poblaciones de menos de 3.000 habitantes, es una cuestión fundamental para terminar con las infecciones de origen hídrico.

f) La socialización del cuidado médico -que es muy distinto a la socialización de la medicina- nos permitirá dominar las enfermedades infectocontagiosas por medio de tres instrumentos de acción: $1^{\circ}$ ) por vía de los centros de salud, o mejor dicho, de las unidades sanitarias, funcionando alrededor de los centros sanitarios de cada provincia; $2^{\circ}$ ) por la creación del "médico de familia", y $3^{\circ}$ ) por la organización del Registro Sanitario de Familia. Nos referiremos brevemente al Registro Sanitario porque los otros dos principios, unidades sanitarias y médico de familia, como formas de acción social de la medicina integral, son bien conocidos de todos ustedes.

El Registro Sanitario de Familias permitiría reunir en un solo legajo los problemas médi$\cos$ y sociales de la comunidad en todos los aspectos vinculados a la salud y a la enfermedad, tomando como unidad fisiopatológica, no al individuo, sino a la familia; solo siendo dueños de esta documentación se podrá luchar simultáneamente con los factores biológicos y con los facto- 
res sociales de la enfermedad. El Régimen Sanitario Familiar permitirá coordinar la acción de la Sanidad propiamente dicha con la medicina preventiva, asistencial, la asistencia social y la previsión social, reuniendo todas las fuerzas y haciéndolas concurrir a la difícil tarea de salvar la personalidad humana, reconstruir y construir nuestra arquitectura biológica. Del mismo modo que se ha organizado un Registro Civil, un Registro Militar, un Registro de Identificación Civil, etc., deberíamos organizar un Registro de la Salud Familiar, donde se consignaría el patrimonio humano de la Nación, que es mucho más importante que el registro de las propiedades y de los bienes materiales del Estado.

\section{LA SALUD COMO FUNDAMENTO DE FELICIDAD}

Al comenzar he dicho que estemos preparados para abordar la medicina por cauces mucho más difíciles. No incurriré en la ilusión de creer que ya hemos triunfado, por la simple comprobación de los grandes triunfos de la epidemiología; esos éxitos, en el conjunto del problema, son todavía pequeños. Tampoco incurriré en el candoroso optimismo de suponer que la cuestión de fondo, que es la salud natural del hombre como fundamento de su felicidad y bienestar, será resuelta mediante costosos hospitales, grandes fábricas de medicamentos o prolija organización de los médicos. El ideal es que el hombre pueda gozar de esa salud natural, como resultante del armónico desarrollo de sus actividades somáticas, mentales y sociales y no de una especie de estado negativo de falta de enfermedad -que es como entendemos hoy los médicos la salud-pues tratamos bajo todos los medios de mantenerla, o recuperarla recurriendo a expedientes más o menos eficaces pero artificiales, con sueros y vacunas, vitaminas y penicilina, hormonas y dietas científicas, exámenes periódicos, médicos y enfermeras. Nos aproximaremos más a la verdad y al verdadero fin de la medicina, cuando todas esas cosas sean menos necesarias. La ciencia médica y la medicina comenzarán a vislumbrar su triunfo el día que debamos cerrar los hospitales por innecesarios y corresponda reducir la actividad de la industria farmacéutica por falta de consumo, y ello ya está ocurriendo en países como Suecia en donde se han clausurado hospitales de tuberculosos por ausencia de enfermos. La esperanza de la humanidad y el objetivo de todos nosotros debe ser precisamente eso, descubrir los secretos de la inmunidad natural, evitar la aparición prematura de las enfermedades degenerativas y mentales, prevenir sus causas y prolongar la vida en condiciones tales de equilibrio orgánico que ésta merezca la pena ser vivida. El nuevo sendero para llegar a esa meta casi utópica parece ser, en este momento, la ordenación de la vida humana dentro de ambientes físicos, biológicos, sociales y económicos que superen las contingencias de la herencia, de la miseria y de la deformación cultural, a menos que venciendo esta especie de medicina dirigida que practicamos ahora, se descubran los misterios del más allá de las estructuras histológicas y fisicoquímicas, de ese más allá de los procesos que conducen a la neurosis, a la locura o al crimen; y si ello ocurriera estaríamos en el camino de la restauración del hombre a la armonía de su ser fisiológico y mental, con lo cual de inmediato se transformaría el universo que no es sino el último resultado de las funciones integrativas de nuestro sistema nervioso y ese universo estaría, recién entonces, constituido para la felicidad del hombre; será el sueño convertido en realidad, aunque el mismo universo siga siendo algo inalcanzable e incognoscible, porque se confunde con Dios y con la eternidad.

\section{FORMA DE CITAR}

Carrillo R. Balance epidemiológico argentino. Salud Colectiva. 2012;8(Supl 1):S93-S99. 